\title{
EVALUACIÓN DE LA HABILIDAD COMPRENSIÓN AUDITIVA DE ESPAÑOL COMO LENGUA MATERNA EN ESTUDIANTES DE EDUCACIÓN SECUNDARIA EN LA CIUDAD DE CONCEPCIÓN
}

\author{
ASSESSMENT OF THE ABILITY OF LISTENING \\ COMPREHENSION IN SPANISH AS MOTHER TONGUE OF \\ SECONDARY STUDENTS IN THE CITY OF CONCEPCIÓN
}

\author{
Monica Tapia-Ladino* \\ Nathalie Ariz Bernales*
}

\begin{abstract}
RESUMEN
A diferencia de las otras tres habilidades (leer, escribir y hablar) escuchar es una actividad permanente, siempre estamos escuchando. La habilidad de comprensión auditiva ha recibido escasa atención por parte de docentes e investigadores por lo cual revisamos el concepto y lo delimitamos de otros con los que tiene estrecha relación: comprensión oral, comprensión de lectura y escucha. A su vez, se presenta los resultados una prueba de comprensión auditiva (PCAELM) cuyo objetivo es evaluar la habilidad respecto de distintos textos orales habituales en los medios de comunicación como son el spot publicitario, la propaganda y la noticia. Para ello se proponen cuatros niveles de comprensión auditiva; un primer nivel, asociado a la comprensión superficial del texto, un segundo y tercer nivel que se relacionan con las inferencias locales y globales respectivamente, así como también un cuarto nivel que corresponde a la interpretación crítica de la información. En la aplicación de la prueba participaron 80 estudiantes de los dos únicos cursos del mismo nivel de secundaria de un establecimiento municipal chileno. Tales resultados indican que el género discursivo no es una variable crucial al momento de comprender un texto, sin embargo, en los niveles de comprensión auditiva propuestos por esta investigación sí se presentan diferencias. Las preguntas asociadas a comprensión superficial y aquellas relacionadas con el propósito del texto son las presentan un mejor desempeño, a diferencia de las relacionadas con la capacidad de realizar inferencias locales y globales.
\end{abstract}

Palabras-clave: Escucha; comprensión auditiva; comprensión oral.

\section{SUMMARY}

As a difference from the other three language skills (reading, writing and speaking) listening is an ongoing activity, we are always listening. The ability of auditory comprehension has

\footnotetext{
* Universidad Católica de la Santísima Concepción, Chile. mtapia@ucsc.cl

** Universidad Católica de la Santísima Concepción, Chile. nariz@magisteredu.ucsc.cl
} 
received scarce attention from teachers and researchers. Therefore, this study revises and delimits this concept from others with which it has close relation: listening comprehension, reading comprehension and listening. In turn, the results of a listening test (PCAELM) are presented, which objective is to evaluate the ability regarding different and common oral texts in the media, like: spots, publicity and news. Four levels of listening comprehension are proposed: a first level associated to the superficial comprehension of the text, a second and third level related to local and global inferences respectively, and also a fourth level which to the critical interpretation of the information. 80 students, of the only two-secondhigh school classes of a public school, participated in the application of the test. The results indicate that the gender discourse is not a crucial variable at the moment of understanding a text, however at the levels of listening comprehension proposed by this research, there is evidence of differences. The questions associated with the superficial level of listening comprehension and those related to the purpose of the text are the one that present a better performance as a difference with the ones related with the capacity to make local and global inferences.

Keywords: Listening; auditory comprehension; oral comprehension.

\section{ANTECEDENTES}

Como se sabe, la oralidad es una actividad natural e inherente al ser humano. Hablar y escuchar son dos caras de la misma moneda que permiten a los hablantes desenvolverse en diversas situaciones cotidianas a lo largo de toda su vida. Saber expresar ideas y saber interpretar las de los otros, influye en el desarrollo de las relaciones interpersonales en la vida personal y profesional.

La comunicación oral que comprende habla y escucha, posee gran importancia en el desarrollo de las actividades cotidianas. De hecho, siguiendo a Cassany (2002) la comunicación oral ocupa un $80 \%$ del tiempo total de los seres humanos, ya sea en periodo de trabajo o de ocio. Asimismo, Rivers y Temperley (1978) realizan un estudio sobre el tiempo que se utiliza en las habilidades lingüísticas de la jornada laboral de un profesional norteamericano. Los resultados indican que la distribución de tiempo es la siguiente: escuchar $45 \%$, hablar $30 \%$, leer $16 \%$ y escribir $9 \%$. Tales datos confirman que las habilidades orales, hablar y escuchar ocupan el $75 \%$ del tiempo, es decir, son mucho más practicadas respecto que las escritas. En esta misma línea, siguiendo a Mendoza (2005) tiene la relevancia de ser transversal a las otras habilidades comunicativas (escribir y leer) por considerarse un medio de adquisición de la lengua.

En la práctica educativa la comunicación oral es la menos abordada, tal vez porque se asume que los estudiantes saben hablar y escuchar (LOMAS, 2013; CASSANY, 2002; LUGARINI, 1996). Por ello, durante muchos años la enseñanza de la lengua se ha enfocado preferentemente a la lectura y escritura. Otra razón que 
podría explicar este fenómeno, es que los profesionales de la docencia han recibido una formación académica insuficiente en cuanto a las habilidades orales (SANZ, 2012; ALFONSO y JELDRES, 1999) a pesar de que estudios como los de Beuchat (1989) y Brown (1980) revelan que la mayor parte del tiempo de un estudiante lo dedica a escuchar. Por su parte, Tapia-Ladino et al (2011) señalan que existe escasa investigación sobre la oralidad en el aula y una creencia generalizada, tanto de profesores como alumnos, que se trata de una habilidad solamente intuitiva.

Ahora bien, la producción oral de textos se desarrolla con frecuencia en actividades como disertaciones o exposiciones, a diferencia de la capacidad de escucha que ha recibido menos atención en términos didácticos y evaluativos (ALFONSO Y JELDRES, 1999). En cambio, en la enseñanza del inglés como segunda lengua, la comprensión auditiva es una de las habilidades más importantes (WILSON y IZQUIERDO 2013, MARTÍNEZ y TAPIA-LADINO, 2011; RICHARDS y LOCKHART, 2002; ROST, 1990). Por ello, se enseña y ejercita explícitamente en conjunto con los otros ámbitos: comprensión de lectura, expresión oral y expresión escrita.

La importancia de este estudio radica en que la comprensión auditiva es unas de habilidades comunicativas más importantes y que menos atención ha recibido en el ámbito educativo. Se espera que los estudiantes sepan extraer ideas principales, inferir e interpretar acerca de los contenidos que los profesores de manera oral. Sin embargo, en qué momento se les ha enseñado a escuchar activamente. Más aún, cuando hacen ingreso a la universidad, se espera que tomen nota autónomamente de las cátedras, no obstante, este estudio deja en evidencia que es una tarea por lograr en la enseñanza escolar. Escuchar activamente es una habilidad que tanto profesores y alumnos se debe entrenar no sólo en el aula, sino que en todas las situaciones comunicacionales que se nos presenta en la vida cotidiana.

\section{FUNDAMENTACIÓN TEÓRICA}

\subsection{Comprensión auditiva desde la perspectiva psicolingüística}

Para Marrero (2001) el proceso de decodificación lingüística se realiza en tres etapas: audición, percepción y comprensión. En la primera fase (audición) se produce la decodificación de la onda sonora del habla como un proceso automático en el cual no interviene la voluntad del sujeto. En una segunda fase (percepción) esas representaciones neurológicas se transforman en unidades lingüísticas, de manera tal que son clasificadas y categorizadas por el sistema nervioso central. 
En una última fase (comprensión) se interpreta el mensaje. Aunque el fenómeno de decodificación parece un proceso simple que relaciona una señal acústica a información semántica es, en definitiva, un fenómeno sumamente complejo que implica tomar decisiones, discriminar e interpretar lo que se oye. Para comprender la complejidad del fenómeno se han propuestos modelos explicativos sobre el proceso de la escucha.

Entre los modelos más influyentes de la comprensión discursiva se encuentra el modelo proposicional (Van Dijk y Kintsch, 1978), que postula que la estructura semántica se organiza en un micronivel (proposiciones individuales y sus respectivas relaciones) y en un macronivel (significado global). De esta manera, el micronivel se construye de manera superficial con el fin de establecer correferencia, en cambio, en el macronivel considera el texto como un todo. Ambos niveles se estructuran y ordenan mediante la aplicación de macrorreglas: omitir, seleccionar, generalizar y construir. La omisión y selección consiste en la supresión de la información irrelevante para elegir aquella que sí lo es. La generalización se basa en la sustitución de varias proposiciones por una general que las represente a todas. Finalmente, la construcción implica el reemplazo de una secuencia de proposiciones por una que las sintetice. En línea con este modelo, el productor del mensaje será capaz de eliminar, interpretar y ordenar la información gracias al conocimiento previo que este posea sobre el discurso.

Otro modelo vigente es el de comprensión e integración (KINTSCH, 1988), el cual propone que la representación de los enunciados se construye a través de dos procesos o fases: comprensión e integración. La fase de comprensión se realiza desde la entrada lingüística (Buttom-up) o de abajo hacia arriba. En esta perspectiva la mente ordena la información, identifica los sonidos, las palabras, las frases, y, por último, analiza el contexto semántico. En cambio, desde el conocimiento del receptor (top-down) o de arriba abajo se da preferencia a la interpretación, esto es, conocimientos previos y socioculturales que tenga sobre el discurso y tipo de texto. La etapa de integración corresponde a la asociación de información mediante inferencias que dependen principalmente del contexto. En la actualidad, Rost (1990) y Flowerdew y Miller (2005) coinciden en que la manera en la que percibimos e interpretamos los mensajes orales es fruto de la combinación de ambos modelos de procesamiento, es decir, top down y Buttom-up. Tales modelos interactúan paralelamente en los niveles fonológico, léxico, sintáctico y pragmático.

Los modelos de Kintsch (1988) y Van Dijk y Kintsch (1978) han servido para dar cuenta sobre cómo se produce el proceso de comprensión que en la práctica funcionan de manera simultánea y coordinada. Un modelo más actual es 
el denominado indexación de eventos (ZWAAN, 1999). Este enfoque postula un análisis de la recepción del mensaje en cinco dimensiones: tiempo, espacio, causa, motivación y protagonista. Si la situación procesada se conecta con tales contenidos o información en la memoria de trabajo, puede establecerse una conexión que se almacena en la memoria a largo plazo. En otros términos, este enfoque considera que cada vez que el individuo comprende el evento o acción se produce una revisión conceptual de tiempo, espacio, causa, motivación y protagonista. El autor a través de este modelo explica cómo se comprende una narración, es decir, una secuencia de acciones ordenadas en el tiempo.

En términos generales, el oyente al realizar el proceso de comprensión auditiva cumple un rol activo en atribuir significado a lo que escucha. A su vez trata de comprender el mensaje, anticipando lo que oirá y tratando de darle una intencionalidad. Con este propósito el oyente activa sus conocimientos previos tanto lingüísticos como no lingüísticos que ha almacenado en su memoria de largo plazo (ALFONSO Y JELDRES, 1998). Por último, la comprensión auditiva permite comprender e interpretar los mensajes con múltiples intenciones y en diversas circunstancias. Es una habilidad que trasciende al ámbito académico, es decir, corresponde a una práctica social que permite satisfacer las necesidades cognitivas, sociales y culturales.

Si bien existe escasa información sobre cómo se podría entrenar la habilidad de comprensión oral en doble dimensión de habla y escucha, los modelos descritos han permitido caracterizar y explicar el proceso de comprensión auditiva. Ahora bien, Behiels (2010) plantea que existe una automatización de la lengua materna, pues no somos conscientes de cómo realizamos esta acción. Por consiguiente, se pueden diferenciar dos tipos de procesos: el automático (secuencias que se activan cuando se presenta una configuración del input particular y no necesitan de la atención activa del sujeto) y el controlado, que ocurre con la atención del sujeto. Los procesos controlados se pueden volver automáticos mediante el entrenamiento. Por lo tanto, es necesario que el oyente sea consciente de las actividades de la comprensión y que se le enseñe de forma explícita a comprender oralmente a través de estrategias. A diferencia del español como lengua materna, en el caso de la enseñanza del inglés como segunda lengua se ha implementado una serie de sugerencias metodológicas y estrategias para desarrollar la habilidad a través de la enseñanza explícita.

\subsection{Comprensión auditiva y comprensión oral}

Los términos de comprensión oral y comprensión auditiva se utilizan indistintamente de modo que a veces suelen confundirse; por ello, proponemos una 
distinción entre ambos conceptos. Por un lado, la comprensión auditiva es definida por el MCER (2002) como el tipo de interpretación en donde el usuario de la lengua recibe y procesa una información de entrada (input) en forma de enunciado. Desde esta mirada, la comprensión es entendida como el acto de recepción y procesamiento de la información a fin de interpretarla. En la enseñanza de segundas de segundas lenguas existe una preferencia por el término comprensión auditiva como se demuestra en los planteamientos de Medina (2002), Córdoba et al (2005), Méndez (2009), Behiels (2010) Gómez et al (2012) y se utiliza preferentemente cuando se trata de interpretar un texto oral asincrónico. Por su parte, King (2007) se refiere al sentido unidireccional de la comprensión aditiva entendida como una transacción no recíproca del lenguaje que generalmente se entrena para dominar una lengua.

Por otro lado, el término comprensión oral puede ser más apropiado dentro del discurso oral dialógico. De manera tal, que el proceso de comprender oralmente se realiza desde la alternancia de hablar y escuchar en donde cobra importancia el lenguaje verbal y no verbal (gestos, posturas, miradas). Y en donde además, los interlocutores ponen en juego su posición social y la motivación que da lugar a la interacción comunicativa. En esta misma línea, Crespo y Manghi (2005) señalan que la comprensión oral se desarrolla dentro de la interacción social y requiere necesariamente una cooperación activa de los participantes.

En definitiva, podemos señalar que la comprensión auditiva suele darse en la comunicación unidireccional para dominar un código lingüístico, en cambio, la comprensión auditiva en la comunicación bidireccional en alternancia de hablar y escuchar.

\subsection{Habilidad de escucha y comprensión auditiva}

Frecuentemente en español se habla de habilidad de escucha en un sentido amplio tanto para comprender textos en sentido unidireccional o bidireccional (LUGARINI, 1996; CASSANY, 2002; PÉREZ FERNÁNDEZ, 2008; COVA, 2012; LOMAS, 2013). La habilidad de escucha más que a la naturaleza temporal o direccionalidad del texto, se refiere al acto de interpretar y la intención que se tiene para ello. Por su parte, Pérez Fernández (2008) prefiere el término escucha comprensiva que significa poner en marcha todas las estrategias necesarias para comprender. Así también, Fonseca (2005) se refiere a esta misma habilidad con el nombre de escucha activa. En definitiva, escuchar activamente o comprensivamente significa tener una disposición psicológica para hacerlo utilizando estrategias y 
habilidades para promover la comunicación efectiva. La escucha activa permite generar un alto grado de fidelidad en la interacción comunicativa.

En cambio como ya habíamos señalado, la comprensión auditiva es un concepto relativamente limitado que suele centrarse en el dominio del código lingüístico a través de textos orales, por lo general, a través de un soporte digital.

\subsection{Comprensión auditiva y comprensión de lectura}

Coincidimos con Buck (2001), Córdoba et al (2005) y King (2007) en que la comprensión auditiva se relaciona estrechamente con la comprensión de lectora, ya que comparten algunas similitudes. Como indican Córdoba et al (2005). En ambas destrezas, se necesita conocer el código lingüístico, la información se procesa desde la mínima unidad hacia el texto completo, se requiere la interpretación del mensaje y el conocimiento previo sobre el tema tratado, se involucra la solución de problemas entre lo que se lee o escucha y el conocimiento previo y se crea una serie de imágenes mentales que reconstruyen el texto (oral u escrito).

Aunque las habilidades de comprensión auditiva y comprensión lectora se parecen en ciertos ámbitos, son destrezas diferentes. Buck (2001) señala que los aspectos de modificación morfológica, prosódicos (acento y entonación) y la velocidad (pausas, dudas), podrían de alguna manera distinguir a la comprensión de lectura de la comprensión auditiva. A su vez, Córdoba et al (2005) establecen diferencias entre ambas habilidades. En primer lugar, el mensaje escuchado posee una naturaleza efímera, en definitiva, no se puede repasar auditivamente el mensaje. En muchos casos, quien escucha se ve obligado a procesar y responder de manera inmediata, en cambio, en la comprensión de textos escritos el lector puede revisar visualmente el texto y releerlo cuantas veces estime conveniente. En segundo lugar, la comprensión auditiva depende principalmente del uso de la memoria, a diferencia de la comprensión de lectura que la requiere en menor medida. En tercer lugar, la comprensión auditiva se puede valer de elementos paraverbales (ritmo, entonación, acento, entre otros) para interpretar el texto oral, así como también formas no gramaticales (repeticiones, pausas y redundancias). Finalmente, la comprensión auditiva requiere de una discriminación de ruidos, esto es, sonidos, música, bocinas, entre otros. Estos elementos pueden enfatizar o afectar el mensaje.

Dado que son escasos los referentes acerca de los niveles de complejidad de la comprensión auditiva, para efectos de esta investigación nos adherimos a la propuesta Pérez (2005) quien sintetiza una serie de modelos que explican los procesos de comprensión por niveles. De esta manera, el primer nivel es de la comprensión literal que consiste en reconocer y recordar. El segundo nivel 
corresponde a la reorganización de la información y se basa en ordenar las ideas mediante clasificación y síntesis. El tercer nivel es la comprensión inferencial en donde el lector hace conjeturas e hipótesis a partir de sus experiencias personales. El cuarto nivel corresponde a la lectura crítica o juicios valorativos y le permite al lector hacer reflexiones sobre el texto. Finalmente, el quinto nivel es de apreciación lectora se refiere al impacto psicológico y estético que produce el texto en el lector.

\subsection{La habilidad de escuchar}

Como sabemos, la competencia comunicativa se entiende como la capacidad de elaborar, producir discursos adecuados, tanto en el ámbito de la oralidad como de la escritura de acuerdo al contexto e incluye cuatro habilidades: hablar, escuchar, leer y escribir; y su adecuación a los diferentes contextos (CASSANY, 2002; MURILLO, 2009; LOMAS, 2013, entre otros). Por su parte, la oralidad comprende dos habilidades: hablar y escuchar. Se trata de un proceso interactivo de las habilidades de producción (hablar) y recepción (escuchar) en un contexto compartido por los interlocutores.

Oír es un fenómeno fisiológico gracias al cual se perciben los estímulos sonoros. En cambio, escuchar requiere una interpretación de lo que se oye (COVA, 2012; ECHEVERRÍA, 2005, 2007). Por lo tanto, sin interpretación, no se produce el fenómeno de escucha. A su vez, interpretar el mensaje se asocia necesariamente a la espera de una respuesta que el interlocutor desea. Por esta misma razón, la escucha es un proceso activo, pues requiere interpretación y comprensión de lo que se escucha (ALFONSO Y JELDRES, 1999; CASSANY, 2002; ECHEVERRÍA, 2007; PÉREZ FERNÁNDEZ, 2008; TAPIA-LADINO et al, 2011). Desde luego, cuando las personas escuchan anticipan el discurso, atribuyen significado a lo que oyen y descubren la intencionalidad de su interlocutor. Una postura más radical es la planteada por Echeverría (2007) quien señala que la escucha es lo que valida el habla, pues ella sólo logra ser efectiva cuando produce en el otro la escucha y el orador espera. Con ello sostiene que la escucha es la competencia más importante en la comunicación humana, ya que sustenta al habla. Por lo tanto, oír es una habilidad innata, podemos oír sin querer hacerlo, sin embargo, escuchar es un proceso interpretativo que requiere de trabajo y práctica. Ahora bien, existen distintas maneras de escuchar de acuerdo a las circunstancias y el contexto en el cual ocurre.

Lugarini (1996) y Lomas (2013) coinciden con Bickel (1982) en que existen cinco modos de escuchar: distraída, atenta, dirigida, creativa y crítica. Según esta clasificación, la escucha distraída es la más superficial de todas. Se trata de un tipo 
de escucha en la que el oyente puede recibir la información de forma distorsionada por causas físicas, psicológicas, pedagógicas o sociales. En cambio, en la escucha atenta existe una motivación del oyente por recibir el mensaje. La escucha dirigida es aquella en la que el oyente reconoce la finalidad de efectuar el proceso, la creativa es aquella donde el oyente dispone cognitivamente de una participación activa, en otras palabras, interactúa la información nueva con la antigua. Y la escucha crítica - la más compleja e incluye todas - opera cuando se tiene conocimiento del tema, es decir, se sintetiza, evalúa e interpreta lo que se escucha para elaborar juicios propios. Aunque esta tipología teóricamente es bastante aceptada, escasean estudios empíricos que la avalen.

Otra distinción la realiza Beuchat (1989), quien señala que existen cinco formas de escuchar: atencional, analítico, crítico, apreciativo y marginal. En el escuchar atencional, el oyente focaliza su atención en un estímulo para obtener información y participar activamente. El escuchar analítico se enfoca en resolver un problema mediante la información recabada. El escuchar crítico se realiza cuando el auditor realiza un juicio del mensaje. Por su parte, el escuchar apreciativo se efectúa con el fin de disfrutar lo que se oye. Finalmente, el escuchar marginal, consiste en captar otros estímulos auditivos cuando el foco de atención está centrado en un elemento específico. Se realiza la tarea de oír mientras se recepcionan otros sonidos. En la primera distinción propuesta por Bickel (1982) se relaciona la habilidad con variados factores como atención, motivación, finalidad y conocimientos previos para llegar a un oyente ideal (escucha crítica). En cambio, la segunda clasificación (BEUCHAT, 1989) apunta al tipo de operación cognitiva que se hace al momento de escuchar.

Otra manera de aproximarse al tema es a través de las etapas que se producen al momento de la escucha. Algunos autores coinciden en que se identifican tres fases en el proceso de la escucha (PAVONI, 1982; LUGARINI 1996; PÉREZ FERNÁNDEZ, 2008; COVA, 2012, LOMAS, 2013). La primera corresponde a la preescucha, que es el momento de planificación (se determina el propósito o los objetivos). Un segundo momento es el proceso de escucha propiamente tal. Por último, la etapa de post-escucha es aquella en donde se evalúa el proceso de comprensión de los enunciados. Estas diferenciaciones permiten enfrentar una didáctica de la escucha.

Ahora bien, cuando se escucha generalmente se tiene un objetivo para hacerlo. Para Mcentee (1999) entre los propósitos se encuentran empatizar, informarse y evaluar. A su vez, Fonseca (2005) agrega a estos: disfrutar y entender. Así también existen algunos factores de carácter afectivos, intelectuales, 
gramaticales, didácticos y programáticos que dificultan la escucha comprensiva son (PÉREZ FERNÁNDEZ, 2008).

Otra dimensión de análisis se relaciona con las microhabilidades que favorecen la capacidad de escuchar. Cassany (2002) indica seis componentes de la habilidad de escuchar: reconocer, seleccionar, interpretar, anticipar, inferir y retener. Para utilizar estas microhabilidades es necesario poseer conocimientos más o menos globales sobre la gramática y el léxico de la lengua, porque nos permite reconocer, segmentar e interpretar los enunciados lingüísticos. Tales microhabilidades no trabajan en un orden determinado, sino más bien interactúan entre sí, al mismo tiempo y en distintos niveles. De esta manera, se anticipa y se infiere información semántica, pero al mismo tiempo discriminamos los sonidos pronunciados asignando un significado. En síntesis, cada estrategia asociada a una microhabilidad tiene por objetivo desarrollar la comprensión oral tal como se realiza en la lectura.

Como hemos revisado, la capacidad de escucha asociada al proceso interactivo de comunicación, se entiende como un fenómeno activo y complejo que ocurre en variados niveles (desde el simple acto de oír hasta interpretar), diversos momentos (antes, durante y después de realizar la acción) y variados propósitos y estrategias.

Por último, tal como hemos señalado nos parece que la habilidad de escucha es entendida en un sentido amplio e incluye la noción de comprensión auditiva. Por lo tanto, para efectos de esta investigación es más preciso utilizar el concepto comprensión auditiva.

\subsection{La evaluación de la comprensión auditiva}

La evaluación de la comprensión auditiva se encuentra contenida en las sugerencias metodológicas de la enseñanza en segundas lenguas que a continuación revisaremos. Un primer alcance respecto a las características de la tarea en la comprensión auditiva, es que la actividad se encuentre orientada hacia un objetivo o meta (BEHIELS, 2010), es decir, se escuche para algo. Por su parte, Nunan (1999) sugiere realizar actividades concretas hacia un objetivo explícito como dar indicaciones o formular preguntas antes y luego de escuchar el texto oral. Otro aspecto relevante es la verosimilitud de la tarea. Algunos autores coinciden que los estudiantes deben enfrentar situaciones de la vida real (ALFONSO y JELDRES, 1999; CASSANY, 2002 y BEHIELS, 2010), puesto que la autenticidad de la actividad permitirá movilizar las estrategias adecuadas para afrontar el reto.

Rost (1990) señala que en las pruebas de comprensión auditiva es indispensable tener cuidado con las instrucciones de las tareas. Una mala instrucción puede afectar el desempeño de la comprensión auditiva. Asimismo, Sherman (1997) y Hugues (1989) coinciden en que las preguntas se deben dar a conocer después de escuchar 
una vez el audio para que los estudiantes se familiaricen con lo preguntado. Luego debieran escucharlo una segunda vez para responder.

En definitiva, el diseño de una actividad orientada a la comprensión auditiva debe considerar una serie de factores metodológicos: las características de la tarea, la calidad de los materiales, los tiempos de aplicación y las instrucciones. Tales aportes entregan sugerencias metodológicas para elaborar actividades en la interrelación del texto oral con la tarea de acuerdo al contexto en el cual se efectúa. Sin embargo, hay que considerar que su principal objetivo es que los estudiantes adquieran una segunda lengua. En español, por el contrario, el desarrollo de la comprensión oral tiene como objetivo entrenar la destreza.

Respecto a la comprensión auditiva en español se destaca el trabajo de Galán (2015) en donde un examen evidencia que de las habilidades comunicativas, la menos desarrollada es la comprensión auditiva.

\section{METODOLOGÍA}

\subsection{Diseño de Investigación}

La presente investigación se aplica una estrategia cuantitativa. Se recolectaron los datos mediante una prueba escrita que permitió medir los niveles de comprensión auditiva en lengua materna de los participantes para describir, y a su vez, evidenciar estadísticamente el fenómeno. A su vez, se basó en un diseño no experimental de tipo transeccional con alcance descriptivo y exploratorio. En definitiva, la investigación constituye un acercamiento a la comprensión auditiva en español como lengua materna.

\subsection{Los participantes}

En esta investigación participaron 80 estudiantes de secundaria de un establecimiento municipal de la comuna de Concepción, Chile. La muestra intencionada se constituyó de los dos únicos cursos del mismo nivel de este establecimiento con el objetivo de describir concretamente esta realidad escolar. La edad de los informantes varió entre los 15 a 18 años.

\subsection{Instrumento}

Con el fin de averiguar cómo es la comprensión auditiva de los estudiantes elaboramos un instrumento de evaluación: Prueba de Comprensión Auditiva de 
Español como Lengua Materna, desde ahora en adelante PCAELM. El diseño del instrumento se llevó a cabo en dos etapas una etapa teórica y otra procedimental. La primera etapa de carácter teórica consistió en la selección y reorganización de referencias teóricas, en primer lugar, de la habilidad de escucha en donde consideramos tipos de escucha según Bickel (1982), formas de escuchar de Beuchat (1989). En segundo lugar, de la comprensión de lectura, consideramos los niveles de comprensión según Pérez (2005) y los niveles de PISA (OECD, 2010). De este modo, se elaboró una tabla de correspondencia entre los tipos de escucha y niveles de comprensión de lectura. Tal decisión metodológica se debió a los escasos antecedentes empíricos que existen sobre la destreza de comprensión auditiva en español como lengua materna y a la correspondencia que es posible realizar entre la habilidad de escucha y la comprensión de lectura. Posteriormente, se estableció niveles de correspondencia entre tipos de escucha y niveles de comprensión de lectura para consensuar

\subsubsection{Niveles de Comprensión auditiva}

La correspondencia entre los tipos de escucha y los niveles de comprensión nos proporcionó 6 niveles en total. El nivel 1 corresponde a la escucha distraída (BICKEL, 1982) y marginal (BEUCHAT, 1989), que se trata más bien una escucha distorsionada y se asocia al mero acto de oír. Para efectos de esta investigación, este nivel fue descartado, pues no alcanza a realizarse en el proceso de una prueba de medición formal. A su vez, el nivel 6 que integra la escucha creativa (BICKEL, 1982), apreciativa de Beuchat (BEUCHAT, 1989) y nivel de comprensión de lectura apreciativo (PÉREZ, 2005) también fue marginado, debido a dificultad de evaluar un nivel de valoración o apreciación en una prueba de medición estándar. En síntesis, la prueba PCAELM fue diseñada sobre la base de los diferentes 4 niveles de comprensión auditiva que se resumen en el siguiente cuadro.

\section{Cuadro 1. Niveles de comprensión auditiva de acuerdo a los criterios de logro}

\begin{tabular}{|l|l|}
\hline Nivel & Criterios de logro \\
\hline $\begin{array}{l}\text { Nivel } 1 \\
\begin{array}{l}\text { Encontrar información explícita en un audio. } \\
\text { (nivel superficial) }\end{array}\end{array}$ & $\begin{array}{l}\text { Focaliza la atención para recuperar información } \\
\text { explícita y reconoce la idea principal de un } \\
\text { fragmento auditivo. }\end{array}$ \\
\hline $\begin{array}{l}\text { Nivel } 2 \\
\text { Encontrar información no explícita en un audio. } \\
\text { (nivel microestructural o local) }\end{array}$ & $\begin{array}{l}\text { Realiza inferencias a partir de la información } \\
\text { explícita de un fragmento auditivo. Se maneja } \\
\text { con la ambigüedad y conceptos abstractos. }\end{array}$ \\
\hline
\end{tabular}




\begin{tabular}{|l|l|}
\hline $\begin{array}{l}\text { Nivel } 3 \\
\text { Establecer relaciones inferenciales y resolver } \\
\text { problemas en un audio. } \\
\text { (nivel macroestructural o global) }\end{array}$ & $\begin{array}{l}\text { Utiliza información explícita y no explícita } \\
\text { para resolver un problema o generar hipótesis. } \\
\text { Comprende globalmente el texto oral. }\end{array}$ \\
\hline $\begin{array}{l}\text { Nivel } 4 \\
\text { Analizar e interpretar críticamente un audio. } \\
\text { (nivel extratextual) }\end{array}$ & $\begin{array}{l}\text { Analiza e interpreta la estructura global de los } \\
\text { textos orales en términos de sus propósito, } \\
\text { tono, estructura y efecto. }\end{array}$ \\
\hline
\end{tabular}

Una segunda etapa procedimental consistió en la selección de los audios reales de acuerdo al propósito del instrumento y factibilidad de evaluación. Los criterios que utilizamos para la elección de los audios fueron los siguientes:

\section{Cuadro 2. criterios para la selección de audios}

\begin{tabular}{|c|c|}
\hline Criterio & Descripción \\
\hline Duración & Entre 30 segundos y 1 minuto y medio \\
\hline Soporte & Textos auditivos radiales, podcats y audios de publicidad televisiva \\
\hline Calidad & Se valoró la calidad de sonido en términos de intensidad y claridad \\
\hline Interlocutores & $\begin{array}{l}\text { Presencia de no más de } 3 \text { interlocutores en cada texto oral. Además, que } \\
\text { los hablantes no fuesen personajes conocidos o polémicos para evitar ideas } \\
\text { preconcebidas que generen empatía o rechazo }\end{array}$ \\
\hline Lenguaje & $\begin{array}{l}\text { Se consideró que los interlocutores tuvieran una variación estándar del } \\
\text { español de Hispanoamérica (Chile- Perú). }\end{array}$ \\
\hline Género & $\begin{array}{l}\text { En este aspecto, la primera decisión del equipo investigativo fue utilizar un } \\
\text { texto oral no literario y de uso cotidiano en los medios de comunicación. } \\
\text { De acuerdo a este criterio, se optó por la noticia, el spot publicitario y la } \\
\text { propaganda. }\end{array}$ \\
\hline
\end{tabular}

Para el diseño de la PCAELM se seleccionaron 9 audios con la siguiente distribución: 3 noticias, 3 spot publicitarios y 3 propagandas. Asimismo, para la elaboración del instrumento decidimos utilizar una prueba de selección múltiple. Las razones de tal elección son de naturaleza práctica: demanda menos tiempo y se evita la expresión, pues no queremos evaluar la producción oral o escrita de los estudiantes, sino que los participantes reconozcan la alternativa más adecuada de acuerdo a los distintos niveles de comprensión auditiva. Respecto a la cantidad de preguntas y al tiempo destinado para resolverlas consideramos dos aspectos, en primer lugar, nos basamos en un modelo de examen de comprensión auditiva de 
español como lengua extranjera (INSTITUTO CERVANTES, 2010). En segundo lugar, los niveles de comprensión auditiva propuestos por esta investigación.

A partir de estas consideraciones, el diseño de la PCAELM se constituyó de 9 audios evaluados por 4 preguntas cada uno, que en definitiva, corresponden a los cuatro niveles de comprensión auditiva. En total fueron 36 preguntas con un tiempo de 45 minutos para responderlas. Respecto a la formulación de las preguntas y alternativas, nos basamos en un criterio de consenso y discusión. Cada investigadora propuso la formulación de preguntas y las respectivas alternativas que luego fueron discutidas y consensuadas por el equipo de investigación. Un último resguardo fue desordenar los audios y las preguntas con el objetivo de evitar la mecanización del proceso de comprensión en textos orales que pertenecen a un mismo género discursivo. Sin embargo, tomamos la decisión de mantener las preguntas de nivel 1 (encontrar información explícita) en primer lugar con el fin facilitar el proceso de recuperación de la información. A su vez, se aleatorizaron las alternativas a modo de prevenir patrones de conductas de las respuestas correctas. Finalmente, elaboramos la instrucción y el objetivo de la prueba que fue respectivamente explicitado en el instrumento.

\subsubsection{Validación del instrumento}

La validación de la PCAELM se realizó a través de un protocolo de Juicio de Pares por parte de cuatro jueces expertos: tres académicas universitarias del área de Lenguaje y una profesora de Lenguaje y Comunicación de secundaria. En el instrumento valoró: la calidad del audio, la relación entre pregunta-respuesta y la correspondencia con el nivel de comprensión sugerido.

Con el fin de facilitar la evaluación se transcribieron los audios a fin de que de los expertos pudieran encontrar la información de manera más expedita. Se les solicitó valorar los tres aspectos señalados en una escala del 1 al 5 para expresar su nivel de acuerdo.

\subsection{Variables}

Considerando los ajustes de los Jueces expertos, las variables de nuestra investigación corresponden a los niveles de comprensión auditiva y los géneros discursivos. En primer lugar, los cuatro niveles de comprensión auditiva que se describen en el presente estudio: nivel 1 (superficial), nivel 2 (microestructural o textual), nivel 3 (macroestructural o global) y nivel 4 (extratextual) En segundo lugar, los géneros discursivos de uso cotidiano en los medios de comunicación de 
masas, en este caso, radio y televisión. Lo anterior es una decisión metodológica basada en la propuesta del MINEDUC (2009) que sugiere la formación de ciudadanos competentes en situaciones comunicativas de la vida real. Por esta razón, las investigadoras han seleccionado tres géneros de uso cotidiano: spot publicitario, propaganda y noticia. De este modo, el spot publicitario es entendido como comercial o anuncio corresponde a una pieza audiovisual de producción ajena al medio e insertada en bloques (VIÑEZ, 2004). A su vez, la propaganda es la transmisión de un mensaje en los medios de comunicación de masas con el objetivo de difundir ideas o persuadir. (BONIN, LORITE Y MALDONADO, 2016). Cabe señalar que el spot publicitario tiene un fin comercial en tanto que la propaganda buscar influir en la conducta de las personas o adherir a una ideología. Por último, para este estudio nos apoyamos en Velásquez et al 2005, quienes señalan que la noticia corresponde a un género periodístico, es un texto oral o escrito que relata en forma detallada un acontecimiento con el objetivo de informar.

\subsection{Recolección de los datos}

Antes de llevar a cabo la aplicación de la PCAELM se gestionó la autorización formal a las autoridades del establecimiento. Al mismo tiempo solicitamos el consentimiento informado de cada uno de los estudiantes quienes accedieron a participar de manera voluntaria. Para motivar su colaboración se les indicó que luego de la aplicación de la prueba se sortearía unos audífonos a modo de estímulo. Los participantes fueron comunicados sobre el objetivo de la investigación y la importancia de su participación. Primeramente, se leyeron las instrucciones en voz alta y se solicitó que no conversaran durante la aplicación de la prueba. Luego, se dio paso a la reproducción de los audios a través de un computador portátil y dos parlantes externos proporcionados por el equipo de investigación. Los participantes tuvieron la posibilidad de escuchar cada texto oral dos veces para responder las preguntas. Cabe señalar que la aplicación del instrumento se realizó para los dos cursos de secundaria durante el mismo día en las dependencias del establecimiento. Asimismo, con el objetivo de realizar esta actividad dispusimos de 45 minutos en el horario del subsector de Lenguaje y Comunicación. De esta manera intentamos simular una situación pedagógica cotidiana para los estudiantes. 


\section{ANÁLISIS DE LOS DATOS}

Antes de tabular los resultados se eliminaron las pruebas que no fueron respondidas para asegurar la total disposición de los participantes para colaborar. Se creó una base de datos en Excel. La primera etapa tuvo por objetivo explorar el comportamiento de los datos recogidos y observar tendencias en los resultados. Este análisis se basó en la identificación de los puntajes promedio considerando las variables de género discursivo y nivel de comprensión auditiva. La segunda etapa se efectuó mediante la aplicación de pruebas estadísticas chi-cuadrado y prueba normal de dos colas para probar la incidencia de la variable de edad y para determinar la existencia de diferencias estadísticamente significativas.

\section{RESULTADOS}

El porcentaje de logro de las 36 preguntas oscila entre el $30 \%$ a un $100 \%$. Se decidió marginar de la muestra las preguntas con un porcentaje de acierto muy alto o bajo, esto es, inferior a $10 \%$ o superior a $90 \%$. Por esta razón cuatro preguntas que obtuvieron más un de un $90 \%(1,2,9$ y 33) fueron suprimidas. El criterio aplicado fue que la preguntas acertadas por más del $90 \%$ corresponden a preguntas extremadamente fáciles o que pudieran contener un error de formulación. Esta decisión se fundamenta en la naturaleza experimental de la prueba PCAELM. Asimismo, no se registraron en la aplicación preguntas extremadamente difíciles. A su vez, se investigó si existe relación entre el porcentaje de logro y el género de los participantes, sin embargo, la muestra que se conformó de 25 mujeres y 55 hombres se consideró desigual para integrar tal variable, dado que el $n$ no es comparable. Además se indagó sobre la relación entre la edad de los informantes y su desempeño, dado que existe una variación de edad entre los 15 a 18 años (con gran cantidad de alumnos que han repetido cursos anteriores). No obstante, el análisis estadístico Chi-Cuadrado arrojó el valor de $\mathrm{x}^{2}=13,03$ (P: 0,05 con 9 gdl), por lo tanto, el porcentaje de logro no depende de la variable edad.

El resultado general considera el total de preguntas tomando en cuenta la eliminación de las antes mencionadas. El puntaje ideal para el instrumento fue de 32 puntos y el puntaje promedio alcanzó los 18,45 puntos, mientras el puntaje más alto fue de 26, el más bajo alcanzó los 10 puntos. El desempeño promedio de todos los participantes alcanzó el 57,8\% de logro. Los datos evidencian que los estudiantes poseen un desempeño bajo en la habilidad de comprensión auditiva, pues más de la mitad comprende parcialmente lo que escucha. Lo anterior se corresponde con los 
últimos resultados de PISA en lectura (MINEDUC, 2009) que revela que 63\% de los estudiantes chilenos no supera el nivel 2 -realización de inferencias menores en el texto escrito- En el gráfico 1 se presenta la dispersión entre los puntajes obtenidos por cada participante. En el eje horizontal se muestra a los sujetos mientras que en el eje vertical se indica el puntaje obtenido por cada uno.

Gráfico 1. Dispersión de los puntajes obtenidos por los participantes

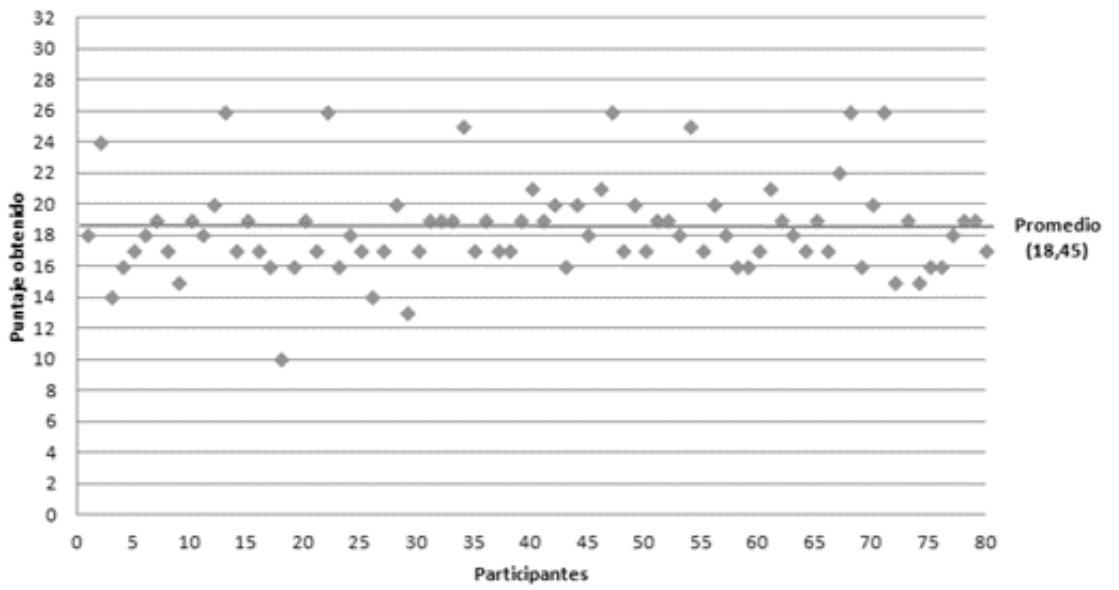

Tal como se aprecia en el gráfico 1, existe una baja dispersión del puntaje obtenido por los participantes en la PCAELM. Se presenta una desviación estándar de 3,04 puntos. De ello se desprende que los participantes se comportan de manera homogénea al momento de enfrentar la tarea de comprender auditivamente. En otros términos, tal habilidad se relaciona escasamente con los rasgos individuales, más bien se trata de un comportamiento uniforme en la muestra. Asimismo, otra forma de aproximarse a los resultados generales es a través de los porcentajes de logro obtenidos en las 32 preguntas que conforman la prueba.

\subsection{Resultados por género discursivo}

Un segundo acercamiento a los datos correspondió al análisis de los géneros discursivos. En el eje horizontal se expone los géneros discursivos: noticia, propaganda y spot, mientras que el eje vertical su porcentaje de logro. 
Gráfico 2. Distribución del nivel logro según variable género discursivo

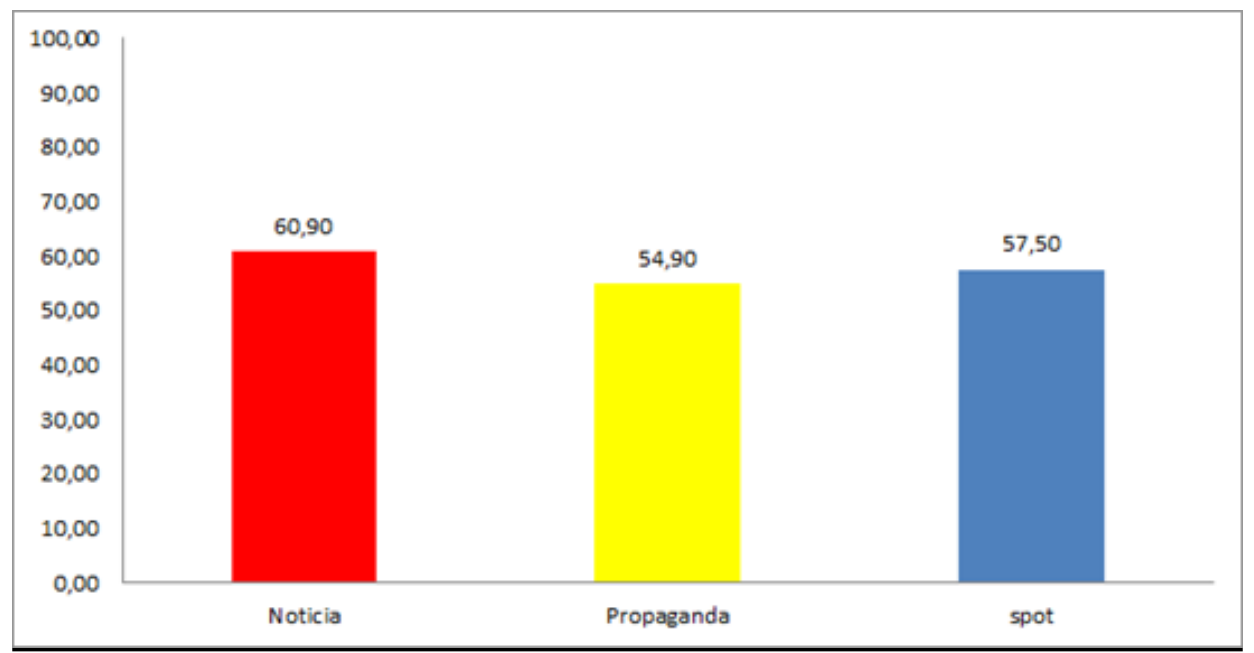

Tal como se evidencia en el gráfico 2, el mejor desempeño de los participantes se produce en textos orales relacionados con la noticia, en donde el porcentaje promedio de logro alcanza un 60,9\%. De ello se desprende que les es más fácil comprender auditivamente preguntas relacionadas con este género, por el contrario, les resulta más dificultoso resolver las que se encuentran asociadas a la propaganda, que obtiene el porcentaje de logro más bajo, un 54,9\%. En términos generales, no se aprecia una gran diferencia entre el rendimiento más alto (noticia) y el bajo (propaganda) sólo alcanza un $6 \%$. A partir de este indicador interpretamos que la variación de género discursivo no genera grandes diferencias en la capacidad de audición, es decir, no divide a los sujetos en términos de desempeño. En sintonía con estos antecedentes, los resultados oficiales de PISA (OECD, 2010) no aluden al género discursivo para evaluar la comprensión de la lectura. Más bien, se distinguen tipos de texto de acuerdo a su propósito principal, entre los cuales se encuentran: la narración, descripción, exposición, argumentación, instrucción y transacción. Sin embargo, los resultados generales (OECD, 2010) no se presentan los desempeños en estas categorías. Un caso distinto corresponde al formato textual, esto es, los textos continuos y discontinuos en los cuales efectivamente se presentan resultados estadísticos en los documentos oficiales. En otras palabras, los informes de PISA restan importancia a los resultados correspondiente a los tipos de textos. Sintetizando entonces, notamos que los tipos de textos y los géneros del discurso no corresponden a una variable crucial al momento de caracterizar el desempeño de los estudiantes en el ámbito de la comprensión, tanto en lectura como en escucha 


\subsection{Resultados por niveles de comprensión auditiva}

Los niveles de comprensión auditiva de español como lengua materna se muestran a continuación en el gráfico 3. En el eje horizontal se indica el nivel de comprensión auditiva mientras que en eje vertical su porcentaje de logro.

Gráfico 3: Distribución de logro de acuerdo a los niveles de comprensión auditiva

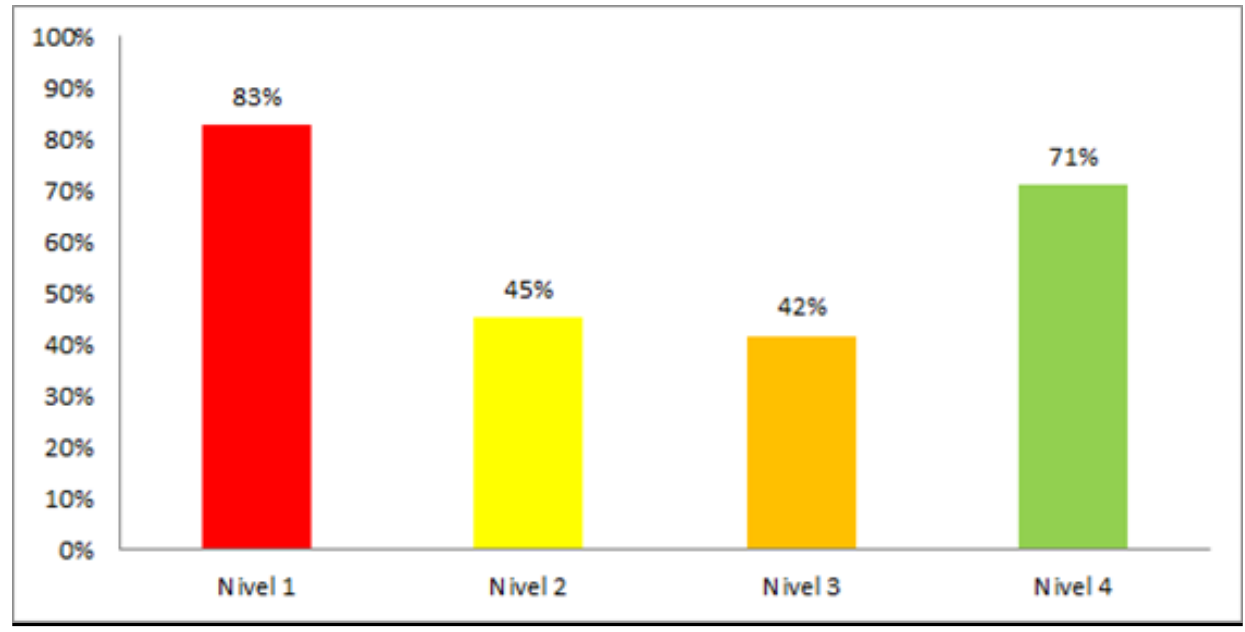

Tal como se aprecia en el gráfico 3, en la variable niveles de comprensión auditiva de español como lengua materna, el mejor logro se obtiene en la comprensión auditiva en el nivel 1, que apunta a la recuperación información. Un segundo mejor desempeño se encuentra en el nivel 4, que consiste en interpretar el propósito del texto oral de acuerdo al género discursivo. Finalmente notamos que los niveles intermedios, es decir, 2 y 3 evidenciaron un comportamiento más bajo y similar entre sí. Mientras que el nivel 2 se refiere a encontrar información no explícita, el nivel 3 se relaciona con formular hipótesis y resolver problemas. Respecto estos niveles intermedios, se efectuó un análisis estadístico para determinar si existía una diferencia significativa entre ellos como se evidencia en la siguiente figura: 


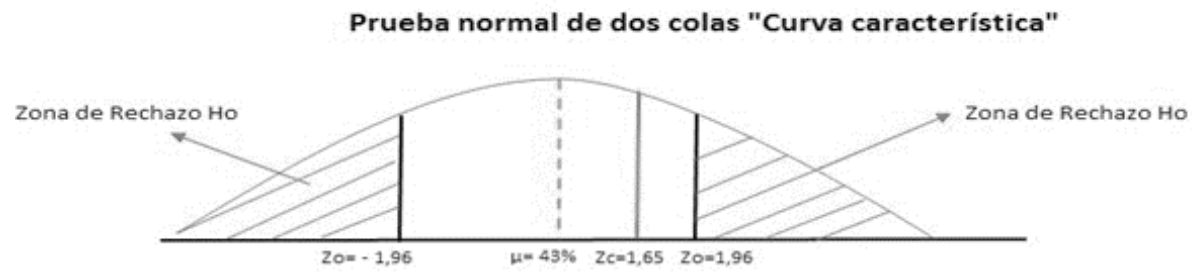

Figura 1. Diferencia de medias a través de la distribución normal

Tal como evidencia la figura 1 la diferencia entre los promedios obtuvo como resultado $Z=1,65$ ( $\mathrm{p}: 0,05$ ), es decir, un resultado inferior a la zona de rechazo. Por lo que se concluye que no se presentan diferencias estadísticamente significativas en tales niveles. Por ello, las preguntas no fueron capaces de generar una discriminación entre los sujetos. Tal comportamiento puede deberse a que los participantes les resulta igual de difícil o fácil responder las preguntas o que ambos niveles se asocian a una misma habilidad. Los niveles 2 y 3 se encuentran asociados a la destreza de realizar inferencias intratextuales de carácter local y global respectivamente. En la propuesta de niveles de comprensión realizada por Pérez (2005) las inferencias corresponden a un solo nivel en donde no se distinguen las de menor y mayor complejidad. Por el contrario, en PISA en la subescala de integración e interpretación, se distinguen seis niveles de inferencias desde las más simples a las más complejas. Los resultados de la PCAELM coinciden con los promedios generales de comprensión de lectura de todos los países de la OECD (2010) en donde el mejor desempeño se encuentra en la habilidad de acceder y extraer información del texto leído (495 puntos), un nivel intermedio lo obtiene la habilidad de reflexionar y evaluar (494 puntos). Finalmente el desempeño más bajo corresponde integrar e interpretar (493 puntos). Cabe señalar que el desempeño de Chile (449 puntos) en comprensión de lectura es inferior al promedio de los países de la OECD (493 puntos). En definitiva, los resultados generales de PISA en lectura se corresponden con los de esta investigación en comprensión auditiva.

\section{DISCUSIÓN}

Los resultados de esta investigación nos han permitido evaluar la comprensión auditiva de español como lengua materna de los participantes mediante la aplicación de una prueba. En cuanto a los resultados generales, los participantes muestran una 
tendencia a responder mejor las preguntas que consisten en extraer información literal. En razón de ello, podemos concluir que los sujetos se caracterizan por una escucha atencional (Beuchat, 1989) que se relaciona con orientar sus esfuerzos para recabar información y Dirigida (Bickel, 1982) en la medida que reconocen para qué lo están haciendo. La variable género discursivo no evidenció grandes diferencias al momento de comprender auditivamente, mientras que la relacionada con nivel de comprensión auditiva asociada a cuatro categorías, sólo logró discriminar tres.

Asimismo, el análisis de datos arrojó un desempeño promedio que alcanza $57 \%$, un resultado bajo considerando que los sujetos pudieron dirigir sus esfuerzos para la realización de la tarea. En primer lugar, tuvieron la posibilidad de escuchar los audios dos veces para asegurar sus respuestas y, en segundo lugar, respondieron preguntas cerradas de selección múltiple. Nos preguntamos entonces ċen el mundo real los estudiantes se encuentran capacitados para comprender auditivamente los distintos textos orales emitidos por los medios de comunicación? Más aún, ćposeen la habilidad de escuchar activamente en la interacción cotidiana con otras personas? La discusión se torna compleja si se piensa que escuchar es una acción que hacemos de manera individual y que muy pocas veces tenemos la posibilidad de saber cómo es. En el marco de estos resultados, coincidimos con Alfonso y Jeldres (1999) en que la comprensión auditiva ha recibido menos atención que las otras habilidades lingüísticas, pues los participantes demostraron encontrarse poco habituados a evaluar su escucha. Téngase en cuenta que un desempeño bajo por parte de los estudiantes no necesariamente se relaciona con la capacidad individual para escuchar, sino también con la escasez de estrategias para hacerlo. En este sentido, damos cabida a los planteamientos de Pérez Fernández (2008) y Fonseca (2005), quienes señalan que la escucha comprensiva o activa no sólo implica el mero acto de interpretar, sino que también contar con una disposición psicológica y poner en marcha diferentes estrategias.

Ahora bien, coincidimos con Buck (2001), Córdoba et al. (2005) y King (2007) en que la comprensión auditiva se relaciona estrechamente con la comprensión de lectura, habilidad que cuenta con un bagaje más amplio de referencias teóricas y empíricas. Por ello, los resultados de este estudio fueron contrastados con los obtenidos en PISA en 2009, año en que el informe de la OECD profundizó en detalle el desempeño de los estudiantes en comprensión de lectura. Nuestros resultados presentan una coherencia con este informe, pues los participantes muestran un bajo nivel de comprensión. El promedio de los estudiantes chilenos alcanza 449 puntos y se encuentra por debajo del promedio de los países de la OECD que bordea los 
493 puntos. En definitiva, interpretamos que la tarea de comprensión les resulta compleja no sólo cuando leen sino también cuando escuchan un texto auditivo.

Los géneros discursivos: noticia, spot y propaganda fueron seleccionados por el equipo investigativo por considerarse recurrentes en los medios de comunicación de masas. Sin embargo, el desempeño de los participantes no mostró una gran variación en este ámbito. Por lo tanto, se desprende que existe una escasa preferencia a la hora de comprender auditivamente un texto. Tal fenómeno podría explicarse por el grado de familiaridad que causan en la vida real estos tres géneros discursivos en los medios de comunicación de masas.

En efecto, el grado de habitualidad se demuestra, por ejemplo, en que las preguntas asociadas a la noticia resultaron más fáciles para los alumnos. En total se realizaron 10 preguntas para esta dimensión, cuyo promedio de respuestas correctas alcanzó un $60,9 \%$. A su vez, la mayor cantidad de acierto se produjo en el nivel 1. De acuerdo a estos antecedentes, podemos inferir que la noticia resulta más fácil para los estudiantes, pues es un género para el cual se encuentran habituados a extraer información explícita en los medios de comunicación. Asimismo, la noticia como género discursivo es abordada en el curriculum de Lenguaje y Comunicación.

A diferencia de los géneros discursivos seleccionados, los niveles de comprensión auditiva propuestos por esta investigación efectivamente mostraron una variación. Los participantes se inclinaron por obtener un mejor logro en las preguntas asociadas al nivel 1 que se relaciona con la extracción de información explícita. Ello podría deberse que la compresión literal resulta menos compleja que otras operaciones cognitivas como inferir o interpretar, es decir, tal nivel sólo requiere recuperar las ideas ya mencionadas.

Otro fenómeno interesante ocurrió en los niveles intermedios 2 y 3 , en donde los estudiantes mostraron dificultad para desenvolverse. Las categorías manifestaron un comportamiento similar entre sí, consecuentemente se demostró a partir de una prueba estadística que ambos niveles se comportan como uno solo. Por consiguiente, la prueba sólo logró discriminar tres categorías. Tal fenómeno tiene bastante sentido si se considera que se basan en la realización de inferencias e interpretaciones a nivel intratexto. En concordancia con tales antecedentes, en los resultados de Informe PISA (OECD, 2010) las tareas asociadas a realizar inferencias reportan más del $50 \%$ de las preguntas y es precisamente en ellas en donde se obtienen los resultados más bajos. En conclusión, los resultados generales de PISA en lectura se corresponden con los de esta investigación en comprensión auditiva. Asimismo, el nivel 4 resultó más fácil que el 2 y 3, situación que puede 
deberse a que los participantes se encontraban habituados a reconocer el propósito de los textos en los medios de comunicación de masas.

\section{REFERENCIAS}

ALFONSO, E. Y JELDRES, V. (1999). ¿Cómo acercarnos a la comprensión auditiva en español? Signos, 32 (45-46), 73-8. Disponible en https://scielo.conicyt.cl/scielo. php? script $=$ sci_arttext\&pid $=$ S0718-09341999000100009

BEHIELS, L. (2010). Estrategias para la comprensión auditiva. Antología de los encuentros internacionales del español como lengua extranjera. 11, 179-194. Disponible en http://www. marcoele.com/descargas/navas/08.behiels.pdf

BEUCHAT, C. (1989). Escuchar: el punto de partida. En Lectura y Vida. Revista latinoamericana (3), 20-25. Disponible en http://www.lecturayvida.fahce.unlp.edu.ar/numeros/ a10n3/10 03 Beuchat.pdf

BONIN, J.; LORITE, N. Y MALDONADO, J. Publicidad, propaganda y diversidades socioculturales. Quito: Ediciones Ciespal.

BICKEL, J. (1982). L`éducazione favorativa. Liorno: Belforte Editore Libraio.

BROWN, G y YULE, G. (1980). Teaching the spoken languaje. Cambridge: Cambridge University Prees,

BUCK, G. (2001). Assessing listening. Cambridge: Cambridge University Press.

CASSANY, D., LUNA, M. y SANZ, G. (2002). Enseñar la lengua. Barcelona: Graó.

CÓRDOBA, P.; COTO, R. y RAMÍREZ, M. (2005). La comprensión auditiva: definición, importancia, características, procesos, materiales y actividades. Actualidades investigativas en educación, 5 (1), 1-15. Disponible en https://revistas.ucr.ac.cr/index. php/aie/article/download/9123/17505

COVA, Y. (2012). La compresión de la escucha. Letras. Vol. 54 (87), 98-109. Disponible en $\quad h t t p: / / w w w . s c i e l o . o r g . v e / s c i e l o . p h p ? s c r i p t=s c i \_a r t t e x t \& p i d=S 0459-$ $12832012000200005 \& \operatorname{lng}=\mathrm{es} \& \mathrm{nrm}=\mathrm{iso}$

CRESPO, N. y MANGHI, D. (2005). Propiedades Cognitivas e intersubjetivas de la comprensión del lenguaje oral: posibles elementos para un modelo. Signos. 38 
(59), 269-285. Disponible en https://scielo.conicyt.cl/scielo.php?script=sci arttext\&pid $=$ S0718-09342005000300001\&lng $=$ es\&nrm $=$ iso

ECHEVERRÍA, R. (2005). Ontología del lenguaje. Santiago de Chile: J.C. Sáez Editor.

ECHEVERRÍA, R. (2007). Actos de lenguaje. Volumen 1: La escucha. Santiago de Chile: J. C. Sáez editor.

FLOWERDEW, J. Y MILLER, L. (2005). Second language listening: theory and practice. Cambridge: Cambridge University Press.

FONSECA, S. (2005). Comunicación Oral: Fundamentos y práctica estratégica. Ciudad de México: Pearson Educación.

GALÁN, R. (2016). Comprensión auditiva: evaluación y aprendizaje. Iztapalapa, (79), 31 46. Disponible en https://dialnet.unirioja.es/descarga/articulo/5716872.pdf

GÓMEZ, L., SANDOVAL, M. y SÁEZ, K. (2012) Comprensión auditiva en inglés como L2: Efecto de la instrucción explícita de estrategias metacognitivas para su desarrollo. Revista de Lingüística Teórica y Aplicada. 50 (1), 69-93. Disponible en https://scielo. conicyt.cl/scielo.php?script $=$ sci_arttext\&pid $=$ S0718-48832012000100004\&lng $=e$ $\mathrm{s} \& \mathrm{nrm}=\mathrm{iso}$

HERNÁNDEZ J., y IZQUIERDO, J. (2016). Metacognition and L2 Listening: Observation of University-level Teaching Practices. Revista electrónica de investigación educativa, 18 (1), 39-52. Disponible en https://www.researchgate.net/publication/302196256_ Metacognition_and_L2_Listening_Observation_of_University-level_Teaching _ Practices

HUGHES, A. (1989). Testing for Language Teachers. Cambridge : CUP.

INSTITUTO CERVANTES (2010). Examen comprensión lectora y auditiva en nivel A2. Disponible en https://examenes.cervantes.es/sites/default/files/dele_a2_2010_1_ cl_ca.pdf

KING, P. (2007). Estudio multidimensional de la oralidad a partir de los textos escolares para la enseñanza del inglés. Signos. 40(63). 101-126.

Disponible en https://scielo.conicyt.cl/scielo.php?script=sci_arttext\&pid $=$ S0718-09342007000100006 
KINTSCH, W. (1988). The role of knowledge in discourse comprehension: A ConstructionIntegration model. Psychological Review, 95, 163-182. Disponible en http:// psycnet.apa.org/fulltext/1988-28529-001.html

LOMAS, C. (2013). ( $2^{\circ}$ edición corregida y actualizada). Enseñar lenguaje para aprender a comunicar (se). Vol I. Bogotá: Cooperativa editorial Magisterio.

LUGARINI, E. (1996). Hablar y escuchar: por una didáctica del saber hablar y del saber escuchar. Signos. Teoría y Práctica de la Educación, 14 (1), 30-51.

Disponible en http://www.quadernsdigitals.net/datos/hemeroteca/r_3/nr_42/a_638/638.html

MARTÍNEZ, P. y TAPIA-LADINO, M. (2011). Las creencias de los docentes y la enseñanza de la comprensión auditiva en una institución de educación superior. En Arancibia et al. Enseñanza y aprendizaje del inglés como lengua extranjera. Aproximaciones desde la investigación. Santiago: Ril editores, Universidad Católica de la Santísima Concepción.

MARRERO, V. (2001). Fonética perceptiva Addenda. Universidad nacional de estudios a distancia. Disponible en http://www.uned.es/451059/Addenda/addenda.PDF

MCENTEE, E. (1999). Comunicación oral para el liderazgo del mundo moderno. México: McGrawHill Interamericana.

MCER (2002). Marco Común Europeo de Referencia para las lenguas: Aprendizaje, Enseñanza y Evaluación. Disponible en https://cvc.cervantes.es/ensenanza/ biblioteca_ele/marco/cvc_mer.pdf

MEDINA, A. (2002). Modelación de la habilidad de comprensión auditiva para la dirección de su aprendizaje en lenguas extranjeras. Contexto educação, 66, 47-55. Disponible en https:// www.revistas.unijui.edu.br/index.php/contextoeducacao/article/view/1176/930

MÉNDEZ, D. (2010). La comprensión auditiva en la enseñanza del español como lengua extranjera. Revista de Semiótica. 16-17, 217-229. Disponible en http://www.adversus. org/indice/nro16-17/articulos/12VIVII-1617.pdf

MENDOZA, A. (2005). Lengua hablada y lengua escrita: la confluencia de dos códigos en el discurso. "La lengua escrita" realizado en IX Simposio internacional de la sociedad española de la didáctica de la lengua y literatura, Logroño, La Rioja.

MINEDUC (2009). Objetivos Fundamentales y Contenidos Mínimos Obligatorios de la Educación Media. Gobierno de Chile. Disponible http://www.agenciaeducacion.cl/wp-content/ uploads/2013/02/Marco-Curricular-y-Actualizacion-2009-I-a-IV-Medio.pdf 
MURILLO, M. (2009). La habilidad de escuchar. Una tarea pendiente en la educación Costarricense. Revista Artes y Letras. Universidad de Costa Rica, 33 (2), 95-131. Disponible en https://revistas.ucr.ac.cr/index.php/kanina/article/download/1079/1140

NUNAN, D. (1999). Second language teaching and learning. Boston: Heinle \& Heinle Publisher.

OECD (2010). PISA 2009 Results. Executive Summary. Disponible en https://www.oecd. org/pisa/pisaproducts/46619703.pdf

PAVONI, M. (1982). Insegnare I'ascolto. En Pozzo (Coord.). Insegnare la lingua. La comprensione del testo. Milán: Ed. Scol. B. Mondadori.

PÉREZ FERNÁNDEZ, C. (2008). Acercamiento a la escucha comprensiva. Revista Iberoamericana de Educación, 45 (2), 1-15. Disponible en https://rieoei.org/historico/ deloslectores/2039Perez.pdf

PÉREZ, M. (2005). Evaluación de la comprensión lectora: Dificultades y limitaciones. Revista de Educación, Número extraordinario, 121-138. Disponible en http://www.ince. mec.es/revistaeducacion/re2005/re2005_10.pdf

RICHARDS, J. y LOCKHART, C. (2002). Reflexive teaching in second classrooms. Melbourne: Cambridge University Press.

RIVERS, W. Y TEMPERLEY, M. (1978). A practical guide to the teaching of english as a second or foreign language. UP: Oxford.

ROST, M. (1990). Listening in Lenguaje learning. London and New York: Longman.

SANZ, G. (2012). Comunicación oral en el aula. Cuadernos de Pedagogía. Barcelona, España: Wolters Kluwer. No. 423, 46-49. Disponible en http://crateru1.educa.aragon.es/ Comunicacion_oral_cuadernos_de_0512.pdf

SHERMAN, J. (1997): The effect of question preview in listening comprehension tests. Language Testing, 14(2), 185-213. Disponible http://journals.sagepub.com/ doi/10.1177/026553229701400204

TAPIA-LADINO, M., FLORES, V., HEEREN, M., JARAMILLO, D., MEDINA, A. y SÁNCHEZ, C. (2011). Comprensión del fenómeno de la escucha en estudiantes de primer año medio de dos colegios particulares subvencionados de la comuna de Concepción. Revista Enunciación, 16 (1), 84-99. Disponible en https://revistas. udistrital.edu.co/ojs/index.php/enunc/article/download/3591/5234 
VAN DIJK, T. Y KINTSCH, W. (1978). Toward a model of text comprehension and production. Psychological Review, 85, 363-394. Disponible en http://www.someya-net. com/01 -Tsuyaku/Reading/vanDijk_Kintsch_Model.pdf

VELÁSQUEZ, C.; GUTIÉRREZ, L.; SALCEDO, A.; EMEY, J. y VALDERRAMA, J. (2005). Manual de géneros periodísticos. Bogotá: Ecoe Ediciones.

VIÑEZ, V. (2004). Comunicación publicitaria de juguetes de televisión. Alicante: Publicaciones Universidad de Alicante.

ZWAAN, R. (1999). Five dimensions of narrative comprehension: The event indexing model. En. S. R. Goldman, A. C. Graesser y P. van den Broek (Eds.). Narrative comprensión, casuality and coherence: Eassys in honor of tom trabasso. New Jersey: lawrence erlbaum associates Publishers.

Recebido: 22/12/2017

Aceito: 18/07/2018 